\title{
Discussion on the System Optimization of the Energy Development Strategy and Plan
}

\begin{abstract}
Energy is an important basis for economic and social development, and is a critical economic sector. Due to the complexity of the energy system, the interactive relationship with economic and social development, and the enormous investment involved, the optimization of the energy system is of great significance. We should make efforts to develop targets and specific approaches for the rational and optimal development of an energy system in order to avoid big losses due to systematic mistakes.
\end{abstract}

Keywords: energy development strategy, planning system, system optimization

\section{The significance of energy system optimi- zation}

As an important economic sector, energy is the foundation for economic and social development. The required investment amount and scale in energy projects has grown continuously in tandem with the increase of China's economic aggregate. Investment on some large scale energy projects has reached tens of billions of Chinese yuan, while investment in the major bases of energy construction can reach hundreds of billions of yuan, and investment on some major energy technology programs adds up to trillions of yuan. The annual investment on China's energy supply system is over two trillion yuan, and expenditures keep growing. The development of an energy system is closely related to the development of energy consumption. On the one hand, energy consumption demand promotes the changes to energy supply systems and facilitates their development. On the other hand, the development of energy supply systems influences or leads the construction and operation of energy consumption systems. It also affects the structure and layout of production capaci-

Manuscript received December 30, 2013; accepted March 30, 2014

Da-di Zhou (西)

Energy Research Institute of the National Development and Reform Commission, Beijing 100038, China

Email: ddzhou@eri.org.cn ty, as well as people's consumption patterns. Production and consumption activities with higher energy intensity are more dependent on energy, and are more directly influenced by the state of the energy system. Whether or not the development of the energy system is rationalized or optimized, it will still have a significant impact on the economy of energy system construction and operation. Hundreds of billions of yuan of can be saved through the rationalization and optimization of energy system development. If the impact of socio-economic systems on energy utilization is taken into consideration, rationalization and optimization of the energy system can bring much more economic and social impact. The optimization of the energy system is extremely important, because of its complexity, its interaction with economic and social development, as well as its huge investment amount. The essence of energy development strategy planning is to set the target and path for the rational and optimal development of energy system, and to avoid great systematic losses causes by systematic errors.

\section{The main levels of energy system optimiza- tion}

Energy is a giant system. Until now, there has been no suitable system optimization tool which covers all of levels of the system. Thus, an energy development strategy to optimize China's energy system should be designed and analyzed with the following considerations.

(1) Putting the energy subsystem into a bigger economic and social framework for discussion. The research emphasizes the interaction between energy and economic and social development, analyzing the objective condition and requirement for energy development during China's modernization with Chinese characteristics. China's economic and social development is a process to explore new industrialization and new social formation. Instead of generic energy demand forecasting, energy system optimization should research the promotion and constraint relation between China's energy development and sustainable development of consumption and production patterns. The target is for China's energy de- 
velopment to effectively support the energy demand of its economic and social development, and facilitate the transformation of its economic development pattern, in order to speed up scientific development. It is hoped that China's energy production and consumption will realize rational, efficient, green and sustainable development, responding to serious challenges such as energy security, resource constraints and ecological protection.

(2) Optimizing energy mix. Optimizing energy mix is not only dependent on different energy resources' availability and cost, but also needs to take into consideration national economic and social development, including among other things, ecological civilization construction.

(3) Optimizing major energy layout. With a vast territory, China's distribution of energy resources is inconsistent with economic development distribution. Energy distribution optimization should consider the relationship between resource distribution and appropriate regional economic development and should consider comparisons between different energy transportation systems. Regional energy development optimization plans should adjust measures to local conditions, and should not be universally applied.

(4) Optimizing each main energy subsystem. Optimization of the energy system will require the optimization of electrical systems, including the power supply and power grid; optimization of the natural gas supply system, its utilization and allocation; and the optimization of city heating (and cooling) supply systems. Among other factors, it will also require the comparison and selection of a centralized energy system or distributed energy system when the proportion of natural gas and renewable energy gradually increases.

(5) Selecting and optimizing major energy technology lines, such as technology lines for nuclear power, clean coal, advanced power generation, renewable energy (including energy storage), natural gas exploitation and utilization (including unconventional gas), etc.

(6) Key developments and technologies related to energy utilization. Besides the areas of industry energy efficiency and environment protection technology that have already received much attention, China should strengthen city building energy consumption efficiency and transportation energy consumption efficiency optimization from a systematic perspective, formulating both development strategies and medium-term to long-term plans.

\section{The methodology of energy system optimi- zation}

Multi-objective is a characteristic of energy system optimization. Single-objective system optimization cannot satisfy even specific large scale energy project. Energy system optimization requires many basic conditions, such as the technical feasibility of each energy subsystem and incorporated energy technologies, resource availability, flow balance among energy subsystems, technical compatibility, transportation conditions, etc. Economic benefit, including output maximization and cost minimization, is the priority of the optimization goals people pursue. However, there are many objects that cannot be directly measured using economic indicators. For example, energy security involves not only resource availability, exploitation, conversion and utilization technologies and their operational conditions, but also involves many geopolitical factors. In many cases, the externality of energy production and consumption are an important optimization objective or constraint condition. Climate change has become an important constraint and driving force for energy development. With the development of China's policy, society, culture and ecological civilization construction, more and more social development goals need to be incorporated into energy development optimization objectives. Energy system optimization should consider the balance of regions, urban and rural development, and concern for low-income groups' energy service demand. At the same time, social cognition and acceptance for some important energy technology and projects have become important external conditions. Among these objectives, some are complementary, but some are contradictory.

The uncertainty of parameters is another characteristic of energy system optimization. The whole system keeps changing. From the perspective of the economy and society, the driving force of energy demand varies in different stages of economic development. Although there is change law, it is still difficult to forecast the time-point and extent of change. The available quantity of energy resources, the progress of technology development, and cost are all dynamic parameters. As a result, to effectively optimize the energy system and formulate an energy strategy, people need to properly account for uncertainty. In using dynamic optimization methods, people should also set alternative plans for different development conditions, and quickly adjust parameters and results when necessary.

Since its economic reform and opening-up, China has reached great achievement in energy system analysis and using system optimization methods. We have introduced many energy system analysis tools, and research institutes have developed system analysis or optimization models, including as the early MARKAL model, EFOM model, MEDEES model, CTS model for coal power transport optimization, INET model by Tsinghua University, among others. Because global climate change has increasingly influenced energy system development, energy and economic system analysis models based on CGE have been the focus of development and application in recent years. The IPAC model system from the Energy Research Institute (ERI) of the National Development and Reform Commission (NDRC) is a good example. Unfortunately, the system analysis tools applied for energy strategy research are not developed well on the whole. One reason is that the development of system analysis tools cannot meet the complicated requirements of energy strategy issues. Another reason is that the changes of Chi- 
na's energy policy-making subjects have restricted the further development of optimization theories and tools. China's system reform in the energy sector has made great progress since its reform and opening-up period. Energy production administrations have been continuously simplified; energy production enterprises have been restructured; competition has been introduced into the market; and many independent market entities have been created. China's state-owned energy enterprises, on the one hand, are the basis of public ownership, taking more social responsibilities; on the other hand, they face tense competition in the global market as well as a diversified ownership environment in the domestic market. Although many social policies have been concerned with enterprise decisions, it is hard to optimize an individual enterprise's energy system based on the overarching industrial perspective, or produce energy analyses and strategies for large-scale systems from a multi-industry perspective. When you don't hold an office, you don't need to consider the policies. Independent social research institutes have a difficult time conducting quantitative analysis for practical application due to the lack of resources, data, and inside information (such as real operation conditions, economic and cost data). Therefore, it is currently a dilemma-energy system optimization and energy strategy development require more advanced methodology and quantitative analysis tools, while our social research capacity is too weak to carry out practical system analysis.

\section{The approach of energy system optimiza- tion}

In China, there are different opinions on how to optimize energy development. One of them is to rely on the market to solve the problem, further breaking the monopoly, introducing foreign and private capital, and pursuing a "survival of the fittest" strategy to get the system optimized. As evidenced by history and issues currently facing countries throughout the world, energy development strategy and system optimization has not been simple. For example, oil, the dominant resource in the world energy sector, has been tightly controlled by developed countries from the beginning. These countries first colonized, then combined oligopoly oil enterprises with power politics, carving up the major market. In spite of business competition, oil magnates coordinate their production to keep prices high. State corporations directly control the majority of oil and gas resources in the world, and regulate the total investment and supply amount, to maintain market balance. Many European countries formulate national energy development strategy directly, using policy instruments to regulate energy development, especially in terms of energy mix. A typical case is nuclear development in France and some European countries, which has been crucially affected by national strategy and policy. The national energy strategy has also played an important role for European countries in importing former Soviet Union natural gas to improve its energy mix. Renewable energy development in the EU has also relied on national strategy and policy in recent years. The United States, an advocate of a free market economy, has never given up national strategy and policy guidance in energy issues. The US Department of Energy has the most employees of any governmental energy department in the world. Apart from 18 thousand employees in the Federal Department of Energy, there are over 20 directly-affiliated, state-run energy research laboratories with 60 thousand to 70 thousand employees. Most of these labs are involved in national energy strategy-making. Besides, each state in the US has its own administration, regulation and supervision institutions for energy policy. Even the US State Department, the department in charge of diplomacy, has recently established an energy policy office to deal with international energy policy issues. Moreover, OECD countries have set up the International Energy Agency (IEA) for energy policy coordination and evaluation. Developed countries' government agencies and authorities almost regularly publish energy strategy, policy or research reports about energy system optimization development. Energy may be one of the sectors with the most government intervention in the world.

Nowadays, energy systems have obvious scale characteristics. New entrants, only with great scale and investment, can access energy systems and have a place. Market competition, if taken as the major mechanism, would be a high-cost approach for energy system optimization. Some important energy industries, such as the electric system (including the power grid and part of the power generation system), natural gas pipe network and end-use supply system, have a technical monopoly. Even though some other energy industries do not have an obvious technical monopoly, it is still difficult to entirely restructure through market competition. For example, oil supply does not have high technical monopoly characteristic, but the resource is not controlled using the socalled free market mechanism. The competition cost of oil's downstream processes such as refining and distribution are very high, so the market is carved up by international oil oligarchs.

Energy system optimization, to a large extent, relies on government and research institutes. Policymaking and implementation can effectively lead and regulate the market. Each country has its own economy, society and resource endowment. The mix is quite varied, so the approach and measure for energy optimization are different. In different development stages, energy system optimization faces different challenges, constraints, and objectives. China could learn from others' experience in efficiency improvement, environmental protection, protection of security interests, and economic effectiveness, to find areas that are held in common. However, China's social institution, development stage and development goals are different from others, so we cannot copy others' energy optimization approaches. China should consider its own optimal energy development strategy and research and develop its own methodology and tools of energy system analysis. 


\section{The challenges of energy development in China-lack of coordination and optimization}

Since reforming and opening up, China's energy development has made tremendous achievements. However, when China's economy enters deep adjustment period, the contradictions accumulated during rapid demand growth period begin to emerge.

There is a phenomenon of excess production capacity in main energy fields in China, but people have different opinions on the current energy situation and development strategy. For example, it has been forecasted many times that there would be power shortages in some areas and some power grids would have higher peak loads. At the same time, the capacity utilization rate for the whole electric system is sharply decreasing. The peak load, in power grids where shortages have been forecasted, reaches only approximately $60 \%$ of all installed capacity. Even though the growth rate of electric consumption is slowing down, some people still think that China's total power installed capacity will double, and the electric system will be in high-speed growth for a long period of time. The development of renewable energy in China reflects the lack of system coordination and optimization. China's wind power manufacturing capacity has far exceeded its domestic demand. Although the domestic installed capacity has increased quickly, and while wind power load time is declining in general, 20 billion $\mathrm{kW} \cdot \mathrm{h}$ wind power has been abandoned. Wind power layout did not consider the technical constraints and costs of long-distance and large-scale transmission. As a result, wind power has failed to replace coal power, and additional larger-scale coal power plants to be built, to support its transmission technically and economically. China's PV power manufacturing capacity has reached more than 20 times of her domestic demand. The excess competition has created tough conditions so that enterprises have had to force prices down for export. New companies that used to have relative high profit margins became a nightmare to many investors in a few short years.

China's coal market is weak in general. To people's surprise, coal prices fall, but some regions insist on constructing big coal bases. A billion tons of coal capacity is going to be launched on the market, and may bring more excess capacity. $\mathrm{PM}_{2.5}$ has caused a large area of dust-haze air pollution, so China's so intensive energy consumption and coal-dominated energy mix are presenting it with challenges. Many regions still have a strong desire for energy consumption growth, including coal consumption. Due to climate change, low-carbon trends have emerged in global energy development, but China has continued to pay attention to utilization of coal, taking a divergent path from the global trend.

Energy investment risk is increasing. The above examples are just some obvious contradictions, among many other problems to be solved.

\section{The emphasis of energy strategy formula- tion and system optimization}

Seeking profit is becoming the main goal of energy sectors, and local governments are very willing to promote energy industries for a higher GDP growth rate. Under the current government performance evaluation system, the expansion competition among energy enterprises is inevitable. For the general interests of national economic and social development, it is very important to transform the energy sector's development and optimize the entire energy system. Due to its scale and complexity, energy system optimization requires better theories and methods. Since time is limited, realistic problems need to be solved as quickly as possible. First, the concept of comprehensive energy system optimization should be determined. People should analyze related system structures and define in a more integrated way the system boundary for major energy investment decisions, which should also contain the subsystems that may have significant a impact on energy system analysis and decision. We had better apply qualitative indicators for comparison and selection, even if quantitative system optimization tools and data are temporarily unavailable. Some regions and industries only consider limited relative system, or advocate partial interests' maximization. Thus, we should especially prevent partial overextend, which may bring systemic discordance, systemic errors and enormous waste. It is very difficult to realize energy system optimization based on current decision-making capacity, but we are able to avoid obvious mistakes caused by narrow system boundary or incomplete consideration. We should gradually approach system optimization and avoiding system boundary faults.

At present, we should focus on the following issues about system optimization.

(1) Energy system optimization should start with a reasonable control on total energy consumption. Observing earlier energy development practices, it can be seen that each energy industry takes its own path of expansion, and neglects system optimization. A reason for this is the overestimation of energy demand and growth rate. Energy forecasting is difficult, especially medium and long term forecast, so errors are inevitable. Most of the forecasts made by foreign institutes are of scenarios analysis. Those forecasts emphasize the uncertainty of bases and premises. As a result, forecasts need to be tracked and adjusted.

There have always been different opinions on China's energy demand forecasts. One is that energy demand is a kind of anticipation of development under economic growth. If there are constraints from resources and environment security, government should guide energy demand and control its growth through policy tools. In the rapid-growth period, we should be concerned with the inevitable changes of demand's driving forces. The subjective wishes of future growth can- 
not substitute the objective market trend.

The other opinion prefers a high-growth prediction as the target of energy development strategy. This forecast can prevent the potential energy shortages, and meet energy sectors' subjective intention of sufficient growth needs. The highgrowth scenario predicts that China's total energy consumption will double. In this case, China's coal consumption may reach near 5 billion tons, and other energy resources, even in a completely free development environment, will not fill the supply gap. Therefore, there is sufficient market space for all kinds of energy resources to develop to their full extent, and they will not substitute each other. In this circumstance, people do not care if energy excess capacity occurs. They believe increased demand will come again at some point, and that it will consume the excess capacity, so the risk of investment too early will be under control. However, previous investments have suffered tremendous losses, and there may be no market for some kinds of energy products over the long term. With the acceleration of China's economic transition, reanalyzing China's energy demand forecast and controlling China's total energy consumption becomes an important premise of energy system optimization improvement. The speed of energy construction should be under control-in advance technology investment is necessary, but excess capacity should to be avoided.

(2) Pay enough attention to the new requirement for energy development raised by ecological civilization construction. Chinese people cannot tolerate the severe air pollution anymore, so dust-haze control has become an important constraint for energy development, and $\mathrm{PM}_{2.5}$ should reach the new air quality standard as quickly as possible. China needs to adjust its energy development strategy and specific targets, accelerating the development of natural gas and hydropower, and promoting nuclear development. China also needs to adjust its policy for non-hydro renewables and expand their utilization. Total coal consumption will necessarily be further restricted. For most of regions, it is the only choice to cut down total coal consumption to control air pollution. For some central and western regions of China, where there has been no severe dust-haze till now, coal development and consumption space is still limited.

Addressing climate change asks for low-carbon energy development, such as much lower energy intensity, and low-carbon energy supplies. Any energy choice, with medium and long term expectations, should consider the problem of carbon emission constraints.

(3) Increase the economic benefits in the energy sector, and promote the transformation of the model of economic development. Since reform and opening-up, China has experienced many changes. A crucial one is the shift from a material-balance oriented principle in a planned economy to the current economic-benefits oriented principle. In recent years, there is a serious misconception that GDP is partially raised by huge investment. A mistaken idea formed that larg- er investments make greater contributions to the economy, and the energy sector has also suffered from it. It is time to adjust China's energy development direction-pursuing economic benefits, especially the energy system's total benefits.

China's energy resources pricing system are influenced by many factors, so there is plenty room of reform and adjustment of the pricing mechanism, price ratio and price structure. On the other hand, in the capital globalization process, the prices of labor and goods further deviate from their real values. The price of resource products and IT products are very high, and the added value of capital in finance and real estate industries are supernormal, while the added value of common labor, as most of the population can be classified, is too low. Additionally, to pursue economic benefits, we should innovate and develop the economic theory of a socialist market economy. We should combine social responsibility with the improvement of energy service's fairness and popularity, including a continuous increase in the labor force's income level. To improve economic performance, we should gain mainly from avoiding investment faults, making full use of existing equipment and capacity, and increasing equipment load rates.

(4) Strengthen adjustment of energy mix. The proportion of coal in China's energy mix is too high. In the past ten years, China's energy has expanded very fast but without clear direct of strategic adjustment, so the reliance on coal rebounded. Many people are not confident about energy mix adjustment, but it will be the highlight of energy strategy in the future. It corresponds with the trends of international energy resource and technology development; satisfies domestic demand of high-quality energy for further urbanization; and responds to the challenges of ecological and low-carbon development. The technology is available. What we need is political determination to make a strategic choice.

(5) Clarify the purpose and direction of reform and opening-up in energy sector. Although the energy sector holds high expectation for further reform and opening-up, different parties have different understandings and claims. Some people think that reform in the energy sector is simply to break the "monopoly", providing more resources and market opportunities to all ownership economies, further diversifying the channels of investment through methods such as the introduction of more foreign capital. However, instead of the aim, reform is the means-the means to emancipate productivity. When making reform plans, we should firstly analyze the major problem of productivity in the energy sector, to find the major adjustments needed in productive relations to emancipate productive forces. "Emancipating the mind and seeking truth from facts" is still the basic starting point of reform and opening-up. The current energy development situation is different from what it was in the 1980s and 1990s. Nowadays, the principal contradictions in the energy sector are neither supply shortage and investment channel 
limitation, nor the investment and construction lag behind demand expansion. More introduction of investment is not the solution to creating sustainable energy development China. Energy system reform needs to promote highly-efficient, green, low-carbon, and sustainable energy development to increase the total energy efficiency and profits by changing the system and mechanism. Reform needs to liberate the pro- ductivity of publicly owned enterprises dominated in energy sector, to relax all kinds of constraints for energy enterprises, and to adjust the market's signaling system. At the same time, energy reform needs to adjust and improve development strategy. Outstanding development planning can avoid ineffective investment and systematic waste. 